\title{
Remote real-time CNC machining for web-based manufacturing
}

\author{
Lihui Wang*, Peter Orban, Andrew Cunningham, Sherman Lang \\ Integrated Manufacturing Technologies Institute, National Research Council of Canada, 800 Collip Circle, London, Ont., Canada N6G $4 X 8$
}

Accepted 24 May 2004

\begin{abstract}
Today's machining shop floors, characterized by large variety of products in small batch sizes, require dynamic control and realtime monitoring capabilities that are responsive and adaptive to the rapid changes of production capability and functionality. It is especially true when the shop floors are combined with the e-manufacturing concept. However, a highly efficient infrastructure that can integrate the pieces of automated equipment together and link them to the e-manufacturing is still missing. The objective of this research is to develop an appropriate methodology with open architecture for real-time monitoring and remote control of networked CNC machines. A framework named Wise-ShopFloor (Web-based-integrated sensor-driven e-ShopFloor) is designed for this purpose. Within the context, this paper presents a new enabling technology to bring traditional CNC machine tools on-line with combined monitoring and control capability. Issues such as architecture design, methodology development, and prototype implementation are addressed through a milling machine case study. It is expected that the developed technology can be readily applied to real shop floor environments with increased productivity, flexibility, and responsiveness.
\end{abstract}

Crown Copyright (C) 2004 Published by Elsevier Ltd. All rights reserved.

Keywords: Web-based manufacturing; Remote control; CNC machining; Java 3D

\section{Introduction}

Machining shop floors characterized by large variety of products in small batch sizes require dynamic control and real-time monitoring capabilities that are responsive and adaptive to the rapid changes of production capability and functionality. It is especially true when new manufacturing systems such as distributed and reconfigurable manufacturing systems are combined with the e-manufacturing concept. In the near future, multiprocessor-embedded open computer numerical control (CNC) controllers and "plug-and-play" smart sensors will be powerful and intelligent enough to handle numerous runtime exceptions and ready to serve raw data during machining operations. However, a highly efficient infrastructure that can integrate the

\footnotetext{
*Corresponding author. Tel.: +1-519-430-7084; fax: 1-519-4307090.

E-mail address: lihui.wang@nrc.gc.ca (L. Wang).
}

pieces of automated equipment together and link them directly to the e-manufacturing is still missing. A new enabling technology is therefore urgently required to bring traditional $\mathrm{CNC}$ machine tools on-line with combined monitoring and control capability. Without it, the advanced factory automation can hardly become practical in the next generation distributed manufacturing shop floor environments.

Targeting this problem, this research is to serve as the glue in factory automation, and to bring intelligent machines, robots and sensors together through an integrated (including the Internet, web, Java, computer graphics, and smart sensors) collaborative environment. Within the environment, machine operators, production engineers or shop floor managers can share real-time data with each other while performing their own duties through this sensor-driven browser-based collaborative environment.

The objective of this research is to develop an appropriate methodology with open architecture for real-time monitoring and remote control of networked 
CNC machines. A framework named Wise-ShopFloor (Web-based-integrated sensor-driven e-ShopFloor) is designed for this purpose. Within the context, this paper presents a new enabling technology that can bring traditional $\mathrm{CNC}$ machine tools on-line with combined monitoring and control capability. Following a brief literature review of related work in Section 2, the concept of the Wise-ShopFloor is introduced in Section 3. Based on the popular VCM (View-Control-Model) design pattern, detailed Wise-ShopFloor architecture is designed and described in Section 4. Other issues such as security and prototype implementation relevant to remote $\mathrm{CNC}$ machining are addressed through a milling machine case study in Section 5. Based on the case study, Section 6 concludes that the developed technology is reliable and can be readily applied to real shop floor environments with increased productivity, flexibility, and responsiveness.

\section{Literature review}

Since 1993 shortly after the debut of the Web, a number of methods and frameworks have been proposed for building web-based collaborative systems. Most of the frameworks are developed for collaborative design, web-based rapid prototyping, project management, and conflict resolutions during collaboration, e.g. WebCADET [1], CyberCut [2], and NegotiationLens [3]. In terms of technologies used in the existing systems, HTML, Java applets, ActiveX, and VRML (Virtual Reality Modeling Language) are widely adopted for developing the client-side user interfaces. At the server side, technologies including JSP (JavaServer Pages), Java Servlets, and XML are quickly obtaining attentions for new system development. To facilitate viable collaborative systems, application servers must engage users in a $3 \mathrm{D}$ graphical interaction in addition to the dialog-like data sharing, because remote users including engineers and managers need active and visual aids to coordinate their efforts in a distributed environment. Today, collaborative manufacturing tops the wish list for many manufacturers in automotive, aerospace, heavy industry, and even the machine tool industry. Unfortunately, most of the existing manufacturing equipment does not have the built-in capability to transmit and receive data. Few of the available webbased systems are designed for shop floor monitoring/ control, remote CNC machining, or for advanced factory automation. Some related works listed below are limited in their functionalities.

Targeting event monitoring, the latest Cimplicity from GE Fanuc Automation (Charlottesville, VA, USA) allows users to view their factory's operational processes through an XML-based WebView screen, including alarms on every Cimplicity system [4]. The FactoryFlow from UGS (Cypress, CA, USA) is an off-line factoryfloor process, planning, and simulation package [5]. By most estimates, the number of $\mathrm{CNC}$ machines capable of linking to the Internet is less than $10 \%$ of the installed base [5]. Seeking the opportunity in linking CNC machines with the Internet, MDSI (Ann Arbor, MI, USA) uses OpenCNC [6], a Windows-based softwareonly machine tool control with real-time database, to automatically collect and publish machine and process data on a network. The OpenCNC module allows manufacturing engineers to access OpenCNC's Significant Events file, which automatically gathers all motion and PLC data in real time to determine why situations occur on the shop floor. In 1999, Hitachi Seiki (Japan) introduced FlexLink [7] to its turning and machining centers. Working together with $P C-D N C$ Plus from Refresh Your Memory (Santa Clara, CA, USA), FlexLink is able to do in-process gauging, machine monitoring, and cycle-time analysis. Since 1998, Mazak (Japan) has operated its high-tech Cyber Factory concept [8] at its headquarters in Oguchi, Japan. The fully networkable Mazatrol Fusion controllers allow Mazak machines to communicate over wireless networks for applications including real-time machine tool monitoring and diagnostics. In addition, Japan-based Mori Seiki introduced a CAPS-NET system that polls machine tools on Ethernet networks at settable time increments, which is usually set at five-second or longer increments, for manufacturing engineers to get updates on machine tools' run-time production [9]. To bring legacy machine tools with only serial ports on-line, eManufacturing Networks Inc. (Burlington, ON, Canada) introduced its ION Universal Interface and CORTEX Gateway [10] to help the old systems go online, and to monitor information flow and the status of the CNC machine tools on the network.

Despite all the accomplishments, the available systems are either for off-line simulation or for monitoring only. Most systems require a specific application to be installed instead of a standard web browser, which reduces a system's portability. Advanced and distributed shop floor monitoring and remote $\mathrm{CNC}$ control remain impractical as web-based applications due to the realtime constraints. To facilitate a viable web-based environment, application servers must engage users in a $3 \mathrm{D}$ graphical interaction in addition to the dialog-like data sharing, because remote users need active and visual supports to coordinate their efforts in a distributed environment. Reducing network traffic and increasing system performance are the major concerns in web-based system developments. Today, shop floor engineers and machine operators still have to meet and respond the challenges for producing highly complex products, especially in very small batches. To be practical, a web-based monitoring and control system should also be efficient and adaptive, and can address 
dynamic issues such as machine breakdown, product changeover, job delay, etc. that happen unpredictably in today's machining shop floors.

\section{A Wise-ShopFloor concept}

The Wise-ShopFloor is designed to provide users with a web-based and sensor-driven intuitive shop floor environment where real-time monitoring and remote control are undertaken. It utilizes the latest Java technologies, including Java 3D and Java Servlets, as enabling technologies for system implementation. Instead of camera images (usually large in data size), a physical device of interest (e.g. a milling machine or a robot) is represented by a scene graph-based Java 3D model in an applet with behavioral control nodes embedded. Once downloaded from an application server, the Java 3D model is rendered by the local CPU and can work on behalf of its remote counterpart showing real behavior for visualization at a client side. It remains alive by connecting with the physical device through low-volume message passing (sensor data and user control commands). The 3D model provides users with increased flexibility for visualization from various perspectives, such as walk-through and fly around that are not possible by using stationary optical cameras; whereas the largely reduced network traffic makes realtime monitoring, remote control, on-line inspection, and collaborative trouble-shooting practical for users on relatively slow hook-ups (e.g. modem connections) through a shared Cyber Workspace [11].

By combining virtual reality models with real devices through synchronized real-time data communications, the Wise-ShopFloor allows engineers and shop floor managers to assure normal shop floor operations and enables web-based trouble-shooting-particularly useful when they are off-site.

Fig. 1 shows how it is linked to a real shop floor. Although the Wise-ShopFloor framework is designed as an alternative of camera-based monitoring systems, an off-the-shelf web-ready camera can easily be switched on remotely to capture unpredictable real scenes for diagnostic purposes, whenever it is needed. In addition to real-time monitoring and control, the framework can also be extended and applied to design verification, remote diagnostics, virtual machining, and augmented virtuality in construction. It is tolerant to hostile, invisible or non-accessible environments (e.g. inside of a nuclear reactor or outside of a space station).

\section{Wise-ShopFloor framework}

The framework is designed to use the popular clientserver architecture and view-control-model (VCM) design pattern with built-in secure session control. Fig. 2 shows the framework in three tiers, which is the enhanced version of that presented at FAIM2002 [12]. Targeting the real-time monitoring and remote $\mathrm{CNC}$ machining, high efficiency for data communication and security issues are carefully examined. The proposed solutions for meeting both the user requirements of rich visual data sharing and the real-time constraints are listed below:

1) using interactive scene graph-based Java 3D models instead of bandwidth-consuming camera images for shop floor visualization;

2) transmitting only the sensor data and control commands between Java 3D models and device controllers for remote monitoring and control;

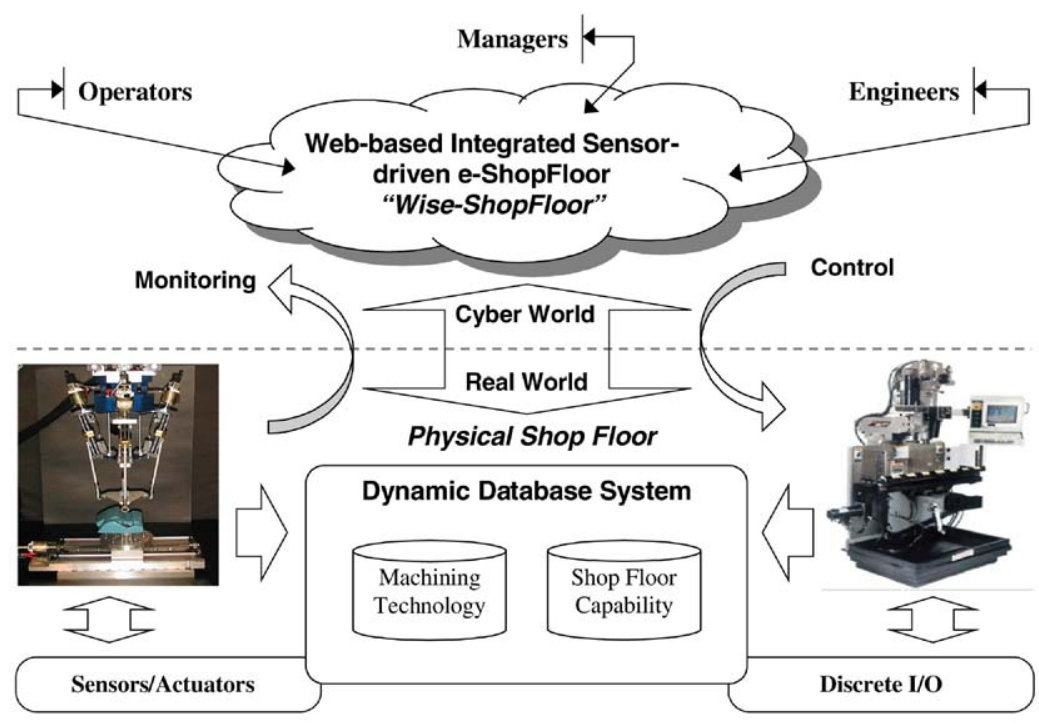

Fig. 1. Concept of Wise-ShopFloor. 


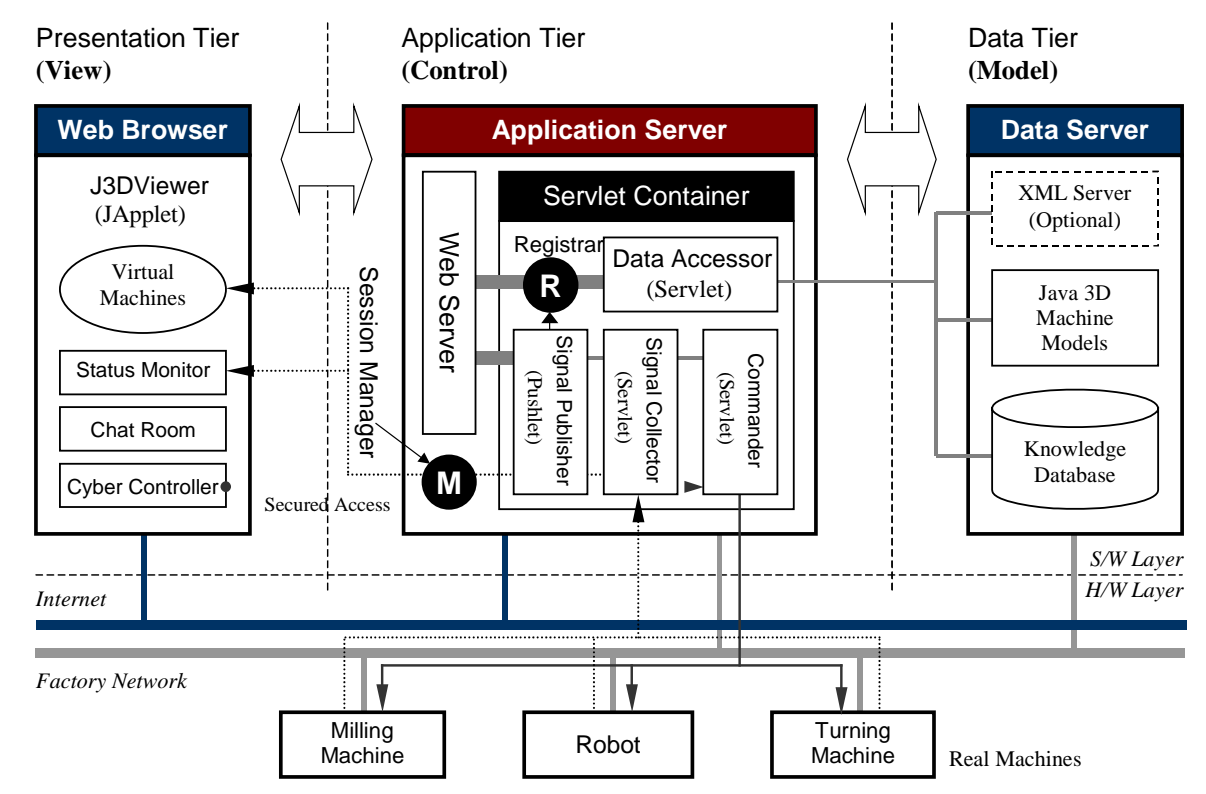

Fig. 2. Framework of Wise-ShopFloor.

3) providing users with thin-client graphical user interface for shop floor navigation; and

4) deploying major control logics in a secure application server.

The mid-tier application server handles major security concerns, such as session control, viewer registration, data collection/distribution, and real device manipulation, etc. A central SessionManager is designed to look after the issues of user authentication, session control, session synchronization, and sensitive data logging. All initial transactions need to pass through the SessionManager for access authorization. In a multi-client environment-the Wise-ShopFloor, different clients may require different sets of sensor data for different models. Constrained by network security, a Java 3D model residing in an applet is not allowed to communicate directly with a real device through socket communication. It is also not efficient to have multiple clients who share the same model talking with the same device at the same time. The publish-subscribe design pattern is adopted to collect and distribute sensor data at the right time to the right client, efficiently. As a server-side module, the SignalCollector is responsible for sensor data collection from networked physical devices. The collected data are then passed to another server-side module SignalPublisher who in turn multicasts the data to the registered subscribers (clients) through applet-servlet communication. A Registrar is designed to maintain a list of subscribers with the requested sensor data. A Java $3 \mathrm{D}$ model thus can communicate indirectly with sensors no matter where the client is, inside a firewall or outside. HTTP Streaming protocol is chosen for the best combination between applets and servlets. For the same security reasons, a physical device is controllable only by the Commander that resides in the application server. Another server-side component called DataAccessor is designed to separate the logical and physical views of data. It encapsulates JDBC (Java Database Connectivity) and SQL queries, and provides standard methods for accessing data (Java 3D models, knowledge base of the devices, or XML documents). The knowledge base is found helpful for device trouble-shooting, while XML will be used for high-level data communication in future extensions.

Although the global behaviors of Java 3D models are controlled by the server based on real-time sensor signals, users still have the flexibility of monitoring the models from different perspectives, such as selecting different 3D machine models, changing viewpoint, and zooming, through J3DViewer at client side. Authorized users can submit control commands through CyberController to the application server. The Commander at server-side then takes over the control for real device manipulations. Another client-side module StatusMonitor can provide end users with a view of run-time status of the controlled device. For the purpose of collaborative trouble-shooting, a ChatRoom is included in the framework for synchronized messaging among connected users.

A proof-of-concept prototype is developed on top of the framework to demonstrate its application in remote monitoring and control. Fig. 3 shows one snapshot of the web user interface of the prototype. A more detailed discussion from device modeling to $\mathrm{CNC}$ control is provided in Section 5 through a milling machine case study. 


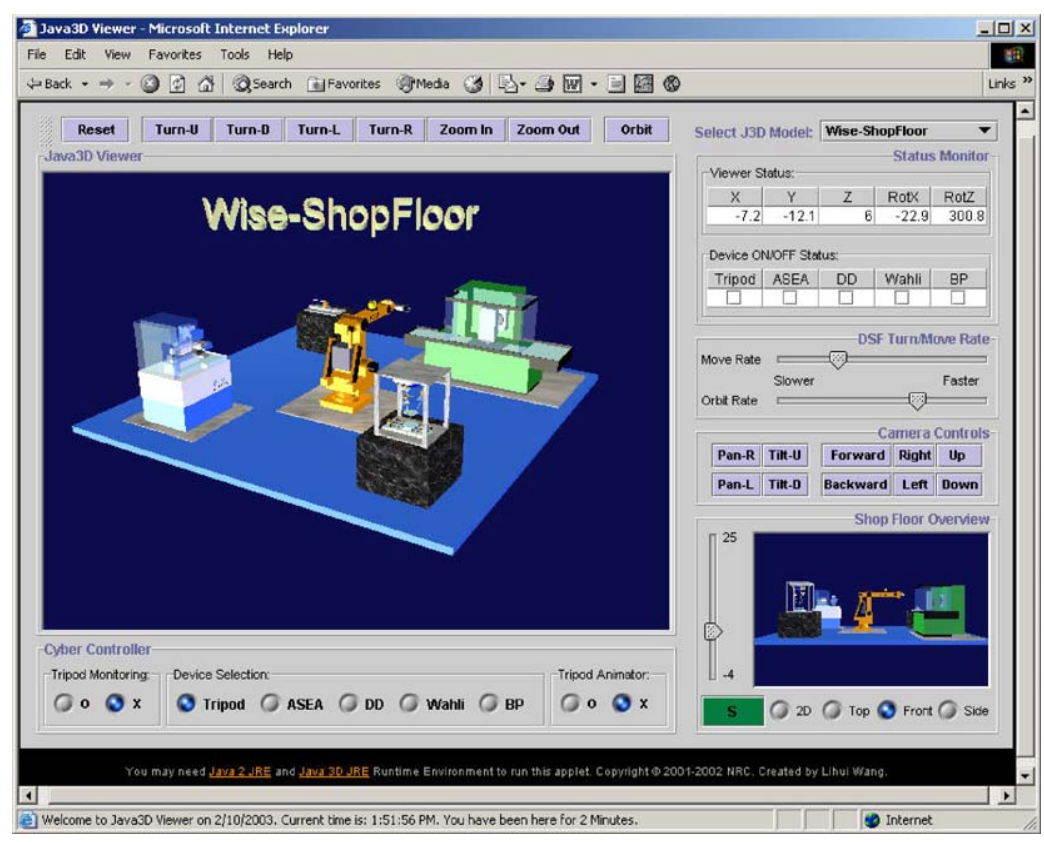

Fig. 3. Web user interface to Wise-ShopFloor.

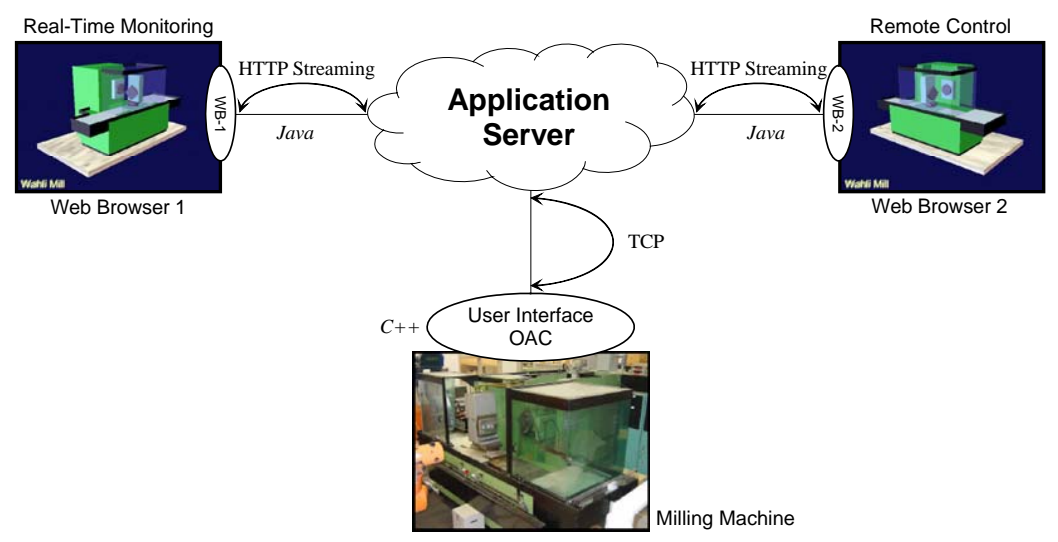

Fig. 4. Test bed configuration.

\section{Remote CNC machining}

\subsection{Test bed configuration}

The test bed used as a case study in this research is a Wahli-51-a 5-axis horizontal milling machine. As shown in Fig. 4, it is equipped with a PC-based OAC (open architecture controller) that serves as a gateway between Wahli and the application server. The Wahli is controlled through a user interface, implemented as a $\mathrm{C}++$ application, running on the same OAC PC, which in turn, beyond a local operator, allows a remote user to send NC (numerical control) line commands to the OAC from a remote web browser and have them executed locally. Remote users can monitor and/or control the Wahli indirectly through the server-side modules (SignalCollector, SignalPublisher, and Commander, as shown in Fig. 2) that perform data collection/distribution and facilitate remote CNC machining. While Java is used for implementing functions in applet and servlets, $\mathrm{C}++$ is selected for local or remote execution control of the OAC. For security reasons, two different communication protocols are adopted for data transmissions at different levels: (1) high-level client-server HTTP Streaming Protocol that is firewall-transparent, and (2) low-level controller-server TCP (Transmission Control Protocol) for better hardware protection. Based on this configuration, it allows a remote user to monitor the absolute and relative motions of all axes as well as to control the spindle speed and feed rate for $\mathrm{CNC}$ machining. 


\subsection{Data collection and distribution}

Java security policy prevents an applet from talking directly with a device through socket communications. In addition, the socket communications have a drawback of inability of traversing firewalls. Clearly, it is important to avoid any security disasters for hardware at shop floor level while developing a web-based system. As mentioned in Section 4, the task of sensor data collection and distribution is handled by a set of serverside modules, such as SignalCollector and SignalPublisher. These modules are developed based on the popular publish-subscribe design pattern. For every client registered with the Registrar (Fig. 2), there is one assigned Subscriber who is knowledgeable of the user's request and responsible of maintaining an active channel between the client and the server. Multi-threading techniques is applied to the module implementations. A comprehensive data flow is given in Fig. 5.

Wahli data collection is accomplished over the TCP connection using a series of 12 floating numbers and one long integer that form one data packet. The format of the packet is defined as follows. receive real-time data anywhere if network connection is provided. In the near future, open-architecture devices (like OpenPLCs and OpenCNCs, etc.) will have web servers and Java virtual machines embedded. This will make the proposed Wise-ShopFloor more practical and efficient in sensor data collection, real-time data transmission, and synchronous communication and collaboration.

\subsection{Remote machine control}

Remote control of the Wahli is possible by sending proper movement commands through the applet-servletcontroller communication illustrated in Fig. 4. In order to remotely control the Wahli, user authentication and control right authorization must be accomplished for the client who requests this function. It is done by setting a bit in the control word in a data packet that is sent to the client. If the client has requested the control right and the bit is set, a message will appear on the screen notifying the user that he/she is now in control of the machine. Fig. 6 shows a web user interface with Wahli selected for monitoring and control.

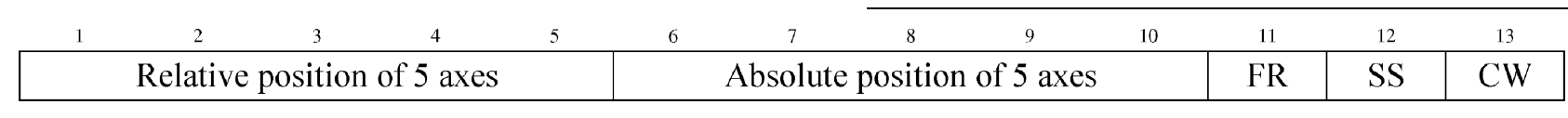

where FR, SS and CW denote feed rate, spindle speed and control word, respectively. A control word is a reserved long integer value indicating the status of the machine, including operation mode, such as manual, auto, or jogging, coordinate system, axis status, etc.

The reason of choosing HTTP streaming as the protocol for data transmission is because it uses standard port 80 and can pass through firewalls. As a result, a shop floor manager or a machine operator can

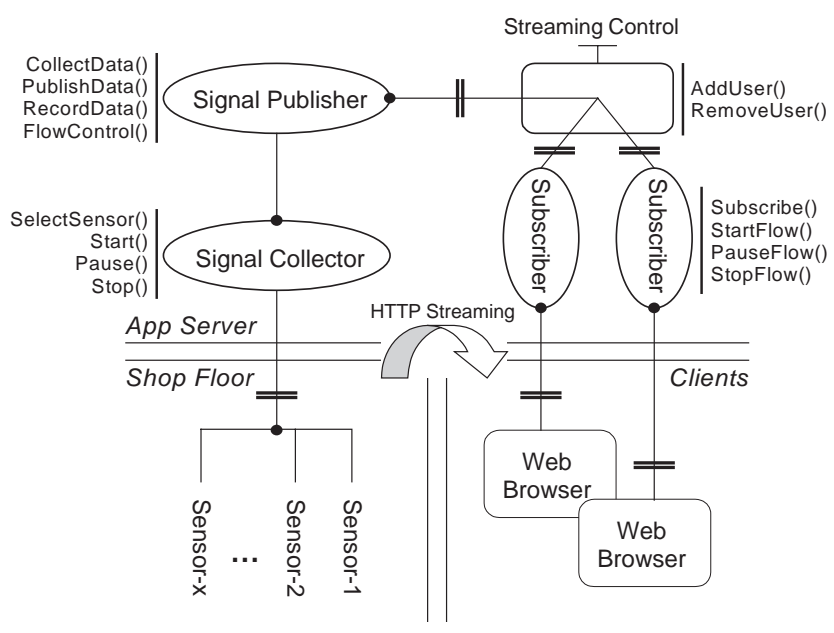

Fig. 5. HTTP streaming-based sensor data collections and distributions.
The motion control of Wahli is accomplished by one of the two methods: jogging control or NC line control. The jogging control is with the use of the individual axis control buttons as labeled in Fig. 6. Each button controls one axis along one direction. For instance, if we want to move the table of Wahli along the $X$-axis in the positive direction, the following must occur.

1. Transmit a default control word from the remote web browser (applet) to the controller-side PC, with the following bit set:

$$
\begin{array}{rcccc}
0 \times 04030040= & 0000 & 0100 & 0000 \\
& A \\
0011 & 0000 & 0000 & 0100 & 0000 \\
\text { BC } & & & D &
\end{array}
$$

A-control request bit,

B-machine-on bit,

C-cycle start bit,

D-jogging mode bit (allowing machine control through jog buttons).

2. Transmit an active control word when the button $\mathrm{X}+$ is pressed. This active control word remains the same until the button is released, at which time it returns to the default control word. The active control word has the following bit set for $\mathrm{X}$-axis 


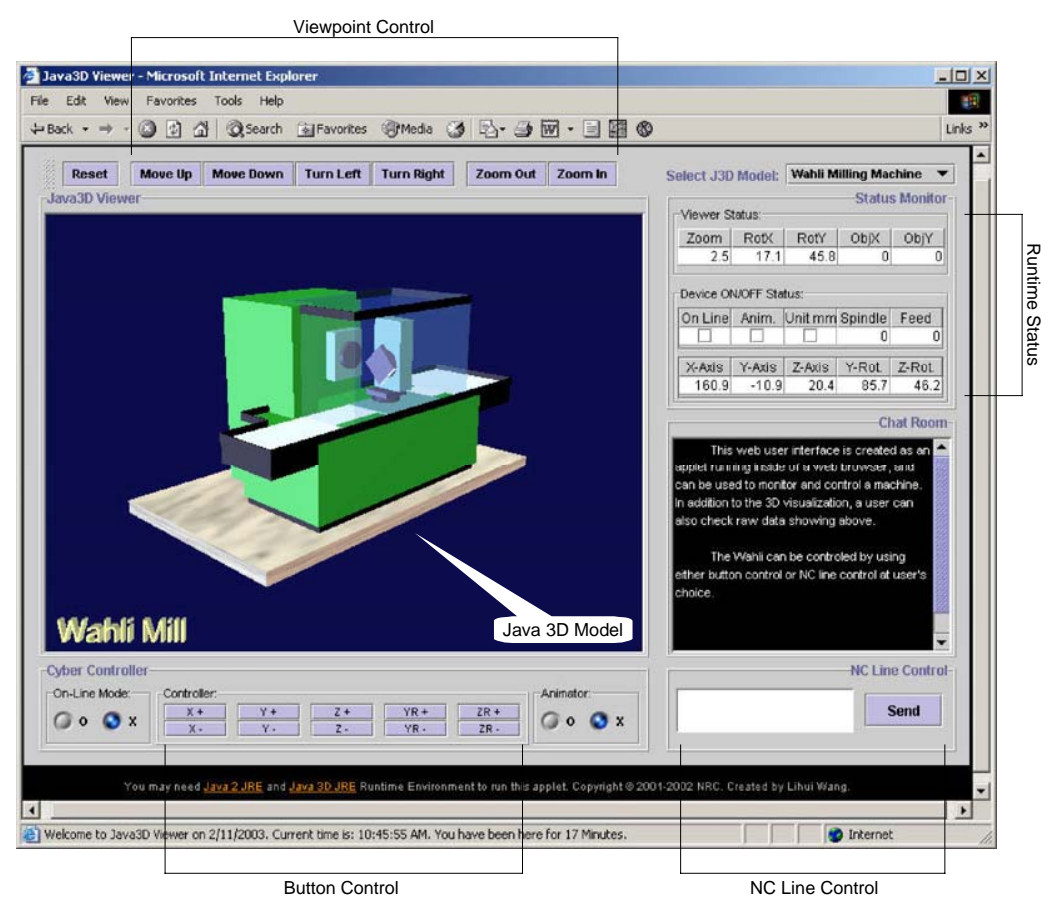

Fig. 6. Web-based real-time monitoring and remote control.

positive button hit.

$\begin{array}{rcccc}0 \times 04430040= & 0000 & 0100 & 0100 & \\ & & A & E & \\ 0011 & 0000 & 0000 & 0100 & 0000 \\ \text { BC } & & & \text { D } & \end{array}$

\section{$\mathrm{E}-X$-axis positive bit.}

Although the button control is straightforward for users who may not be knowledgeable of $\mathrm{NC}$ language, it cannot be used during the actual $\mathrm{NC}$ machining due to its limitations of single-axis control and non-speed control. Instead, the NC line control is recommended. This method sends individual NC lines directly to the Wahli for execution. An experienced remote user can type in one $\mathrm{NC}$ command line and send it as a block to the Wahli controller. For example, the following NC line tells the Wahli to proceed from the current position to the next, incrementally by $(20,-30,10)$ in linear rapid traverse mode. At the same time, the Wahli controller sets the spindle speed to $3000 \mathrm{rpm}$ and turns the flood coolant on.

G0 $\quad \mathrm{X}+20 \quad \mathrm{Y}-30 \quad \mathrm{Z}+10 \quad \mathrm{~S} 3000 \quad$ M8

Using the NC line control, the remote user can send not only individual lines of $\mathrm{NC}$ code but an entire $\mathrm{NC}$ program line by line or in a batch. This allows the client to demonstrate full-scale NC machining and test new programs on the machine from a remote location.
However, for security reasons, $\mathrm{NC}$ code parsing is accomplished in the controller user interface to check the syntax and validity of any $\mathrm{NC}$ lines entered into the text box and sent to the OAC controller.

\subsection{Java $3 D$ visualization}

For the sake of network bandwidth conservation, Java 3D technology is introduced as an alternative of camera-based solution for web-based visualization. Java $3 \mathrm{D}$ is designed to be a mid- to high-level fourthgeneration 3D API [13]. What sets a fourth-generation API apart from its predecessors is the use of scene-graph architecture for organizing graphical objects in the virtual 3D world. Unlike the display lists used by the third-generation APIs (such as VRML, OpenInventor, and OpenGL), scene graphs can isolate rending details from users while offering opportunities for more flexible and efficient rendering. Enabled by the scene-graph architecture, Java 3D provides an abstract, interactive imaging model for behavior control of 3D objects. Because Java 3D is part of the Java pantheon, it assures users ready access to a wide array of applications and network support functionalities [14]. Java 3D differs from other scene graph-based systems in that scene graphs may not contain cycles. Thus, a Java 3D scene graph is a directed acyclic graph. The individual connections between Java 3D nodes are always forming a direct relationship: parent to child.

Utilizing the Java 3D technology, a machine of interest (Wahli in this case) can be modeled as a scene 
graph, representing its physical counterpart in the WiseShopFloor environment. Wrapped in an applet, the model only needs to be downloaded once from its application server, and remains alive through appletservlet communications. The data transmitting through the network are limited to the data showing runtime status of the machine (position, orientation, feed rate, spindle speed, etc.). Driven by the real sensor data, the Java 3D model can demonstrate the true behavior of the real machine with largely improved network performance.

Wahli is a 5-axis horizontal milling machine that requires linear motion control of $X$-, $Y$-, and $Z$-axis, as well as rotary motion control of $B$ - and $C$-axis (around $Y$ - and $Z$-axis, respectively). A combined rotary stage having two rotary motions is mounted on top of an Xtable, whereas the spindle head of the Wahli provides the other two linear motions along $Y$ - and $Z$-axis. Fig. 7 illustrates the Java 3D scene graph architecture of the Wahli.

The scene graph contains a complete description of the entire scene. It includes the geometry data, the attribute information, and the viewing information needed to render the scene from a particular point of view. All Java 3D scene graphs must connect to a Virtual Universe object to be displayed. The Virtual Universe object provides grounding for the entire scene. A scene graph itself, however, starts with BranchGroup (BG) nodes (although only one BG node in this case). A BranchGroup node serves as the root of a sub-graph, or branch graph, of the scene graph. The TransformGroup nodes inside of a branch graph specify the position, the orientation, and the scale of the geometric objects in the virtual universe. Each geometric object consists of a Geometry object, an Appearance object, or both. The
Geometry object describes the geometric shape of a 3D object. The Appearance object describes the appearance of the geometry (color, texture, material reflection characteristics, etc.). The behavior of the Wahli model is controlled by Behavior nodes, which contain userdefined control codes and state variables. Sensor data processing can be embedded into the codes for remote monitoring. Once applied to a TransformGroup node, the so-defined behavior control affects all the descending nodes. In this case study, the 5-axis motions (X-Table, Rotary Stage-1, Rotary Stage-2, Spindle Head, and Spindle) are controlled by their corresponding behavior control nodes, for both on-line monitoring/control and off-line simulation. As the Java 3D model is connected with its physical counterpart through the control nodes by low-volume message passing (real-time sensor signals and control commands, etc.), it becomes possible to remotely manipulate the real Wahli through its Java 3D model.

\subsection{Security of remote machining}

According to an NCMS report [15], there is a growing consensus that linking shop floor hardware to the Internet will become the backbone technology for collaborative manufacturing. However, a major concern of implementing Internet or Web-based systems is the assurance that proprietary information about the intellectual property owned by the organization or information about the company's operations is available only to authorized individuals. In a highly competitive manufacturing environment, the information about the operations of or the information provided by individuals or organizations should only be shared by those involved. Clearly, it is also important to avoid security

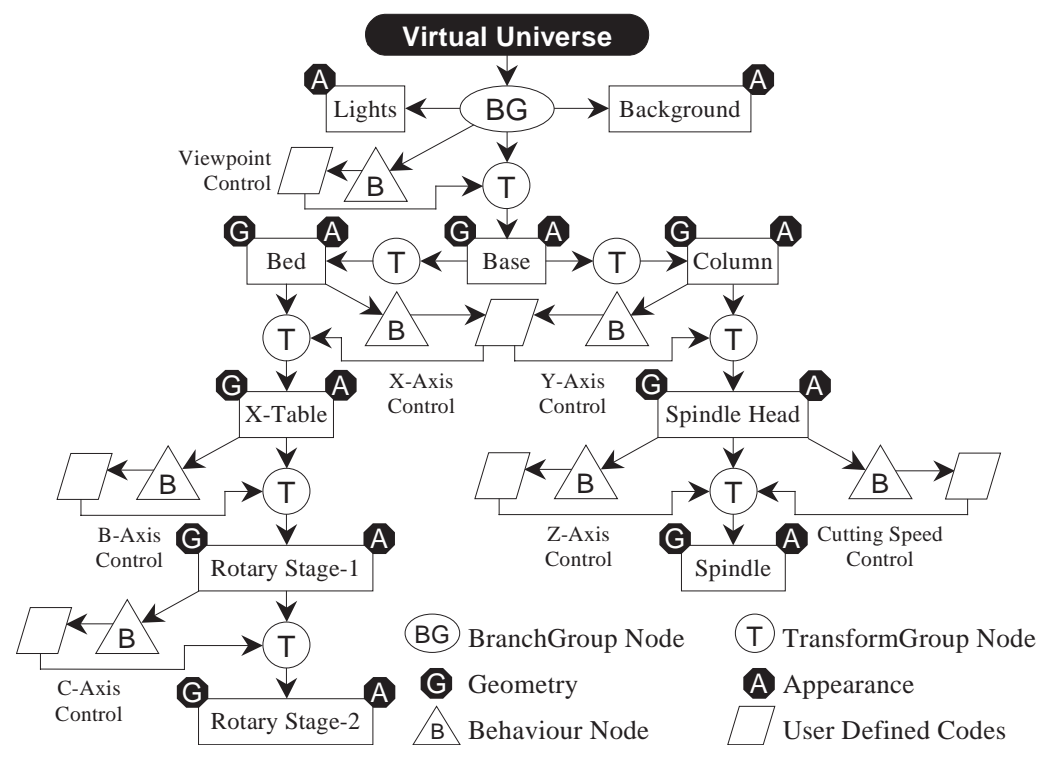

Fig. 7. Java 3D scene graph architecture of Wahli. 
disasters for hardware at shop floor level. Web-based remote monitoring and control typically involve sharing information in the form of detailed run-time operations, as well as real-time and mission-critical hardware controls. For general acceptance of the Wise-ShopFloor concept, the secrecy of the proprietary information must be properly maintained. Our approach to remote CNC machining depends on the familiar security infrastructure embedded in the Java platform. This security architecture consists of byte-code verification, security policies, permissions, and protection domains. In addition to the security infrastructure, other security and privacy issues are considered in the framework for implementation, including digital rights management for information access and sharing, data encryption, and process confidentiality protection.

As shown in Fig. 2, a remote end user can only get indirect access to a machine (Wahli) through the serverside modules, without violating shop floor security. All data communication between the end user and the Wahli goes through the application server, and is processed by a server-side module before passing the data onto its receiver. Only the server-side modules are allowed to collect sensor data or operate the Wahli within their limits of authority. On the other hand, all end users are physically separated from the real shop floor by using segmented networks (Intranet/Internet, and Factory Network) with the application server as a gateway. If further equipped with a highly reliable $\mathrm{NC}$ code parser, our approach to remote real-time $\mathrm{CNC}$ machining becomes possible and practical.

\section{Conclusions}

This paper presents a novel approach to remote realtime CNC machining. It is implemented as a web-based system on top of the Wise-ShopFloor framework with a three-tier architecture. The ultimate goal of our combined web-based and sensor-driven approach is to reduce network traffic by using Java 3D models, while still providing remote users with intuitive environments. Participating in the Java 3D-enabled Wise-ShopFloor, users not only can feel largely reduced network traffic by real-time interactions, but also can obtain more flexible control of the real shop equipment. A milling machine case study demonstrates its feasibility and shows the promise of this approach to the emerging distributed manufacturing paradigm. As decentralization of business grows, a large application potential of this research is anticipated. It can be applied to rapid fabrication, offsite NC machining training, and distant trouble-shooting, in addition to remote real-time monitoring and control.

\section{References}

[1] Caldwell N.H.M., Rodgers P.A. WebCADET: facilitating distributed design support. IEE Colloquium on Web-based Knowledge Servers UK. vol. 9. 1998. p.1-4.

[2] Smith CS, Wright PK. CyberCut: A world wide web based designto-fabrication tool. J Manuf Systems 1996;15(6):432-42.

[3] Adelson B. Developing strategic alliances: a framework for collaborative negotiation in design. Res Eng Des 1999;11:133-44.

[4] http://www.gefanuc.com/products/software/cimplicity_home.asp.

[5] Waurzyniak P. Electronic intelligence in manufacturing. SME Manuf Eng 2001;3:44-67.

[6] http://www.mdsi2.com/products/opencnc.htm.

[7] http://www.hitachiseikiusa.com/tradeshows/Westec/flexlink.html.

[8] http://www.mazak.co.jp/English/sMT\&IT/frame2-factory.html.

[9] http://www.moriseiki.co.jp/product/software/capsnet_e.html.

[10] http://www.e-manufacturing.com/.

[11] Wang L, Wong B, Shen W, Lang S. Java 3D enabled cyber workspace. Commun ACM 2002;45(11):45-9.

[12] Wang L, Sams R, Verner M, Xi F. Web-based and sensor-driven device monitoring and control using Java 3D. In: Proceedings of the 12th International Conference on Flexible Automation and Intelligent Manufacturing, 2002. p. 772-81.

[13] Barrilleaux J. 3D user interfaces with Java 3D. Greenwich, USA: Manning Publications Co.; 2001.

[14] Sowizral H, Rushforth K, Deering M. The Java 3D API specification. Boston: Addison-Wesley; 2001.

[15] NCMS. Factory-floor Internet: promising new technology or looming security disaster. Manufacturing in depth, National Center for Manufacturing Sciences, 2001. 